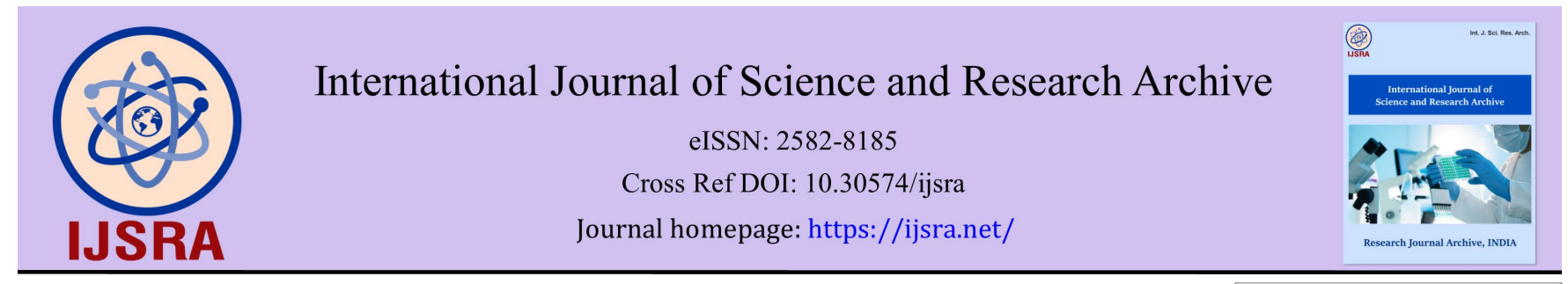

(CASE REPORT)

\title{
Basal cell carcinoma of the antihelix- reconstruction using a pre-auricular flap
}

\author{
Athina Zarachi 1, *, Angelos Liontos 2, Zoi Evangelou 3, Aikaterini Lianou 1, Stefania Gkoura 4, Ioannis \\ Kastanioudakis ${ }^{1}$ and Ioannis Komnos ${ }^{1}$ \\ ${ }^{1}$ Department of Otorhinolaryngology, Head and Neck Surgery, Faculty of Medicine, School of Health Sciences, University \\ of Ioannina, Ioannina, Greece. \\ ${ }^{2}$ Faculty of Medicine, Department of Internal Medicine, School of Health Sciences, University of Ioannina, Ioannina, \\ Greece. \\ ${ }^{3}$ Department of Pathology, Faculty of Medicine, School of Health Sciences, University of Ioannina, Ioannina, Greece. \\ ${ }^{4}$ Department of Oncology, Faculty of Medicine, School of Health Sciences, University of Ioannina, Ioannina, Greece.
}

International Journal of Science and Research Archive, 2021, 03(02), 049-052

Publication history: Received on 14 August 2021; revised on 17 September 2021; accepted on 19 September 2021

Article DOI: https://doi.org/10.30574/ijsra.2021.3.2.0133

\begin{abstract}
We present the case of an 80-year-old female patient with a lesion on the right antihelix of the pinna that examined in the outpatient ENT Department of our hospital. A surgical excision of the lesion was performed, under local anesthesia. We used a superficial based pre- auricular flap to reconstruct the defect. The final postoperative result was satisfactory. The histological examination revealed a basal cell carcinoma.
\end{abstract}

Keywords: Preauricular flap; Basal cell carcinoma; Pinna; auricle; Reconstruction

\section{Introduction}

The auricle is a common area for skin carcinoma development. Basal cell carcinoma (BCC) and squamous cell carcinoma (SCC) are the most common skin tumors occurring in a single anatomic area of the pinna[1, 2] . Comparing the incidence of BCC and SCC in the head and neck skin area with special reference to the pinna, the overall ratio of BCC to SCC remained four to one [3]. Several methods of reconstruction for pinna defects after tumor excision are proposed. Healing by secondary intension, skin grafts, the helix rim advancement technique, the trap door flap, the revolving door flap, the post- auricular island flap, the post- auricular transposition flap, the pre-auricular flap, are only some of the most usual reconstruction techniques that have special indications depending on the region of the auricular lesion[4-10]. We present the case of a woman with a BCC of the right antihelix that removed using a surgical excision. The reconstruction of the pinna was performed using a pre- auricular flap.

\section{Case report}

A 80-year-old female patient examined in the outpatient ENT Department of our hospital, because of a lesion on the antihelix of the right pinna, for about one year (Figure 1). According to the patient's medical history, she did not receive any medication. Through clinical examination, the otoscopy of the right ear did not reveal any pathological findings. No lymph nodes were found in the postauricular, parotid, periparotid and neck area.

\footnotetext{
* Corresponding author: Athina Zarachi

Department of Otorhinolaryngology, Head and Neck Surgery, Faculty of Medicine, School of Health Sciences, University of Ioannina, Ioannina, Greece.

Copyright (C) 2021 Author(s) retain the copyright of this article. This article is published under the terms of the Creative Commons Attribution Liscense 4.0.
} 


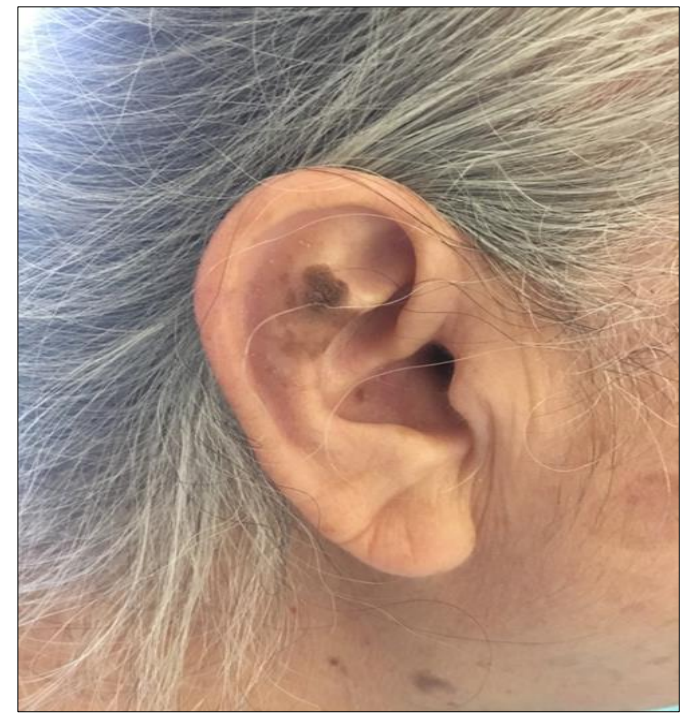

Figure 1 Lesion on the antihelix of the right pinna

After a preoperative evaluation, included hematological and cardiological tests, a surgical excision of the lesion was performed, under local anesthesia. A resection line was drawn around the lesion. A second resection line was drawn in the pre-auricular area, to be used for the reconstructive flap. Neutralized lidocaine with epinephrine used for local anesthesia. The lesion and the perichordrium removed through an excision. A central part of the cartilage was also removed (Figure 2).

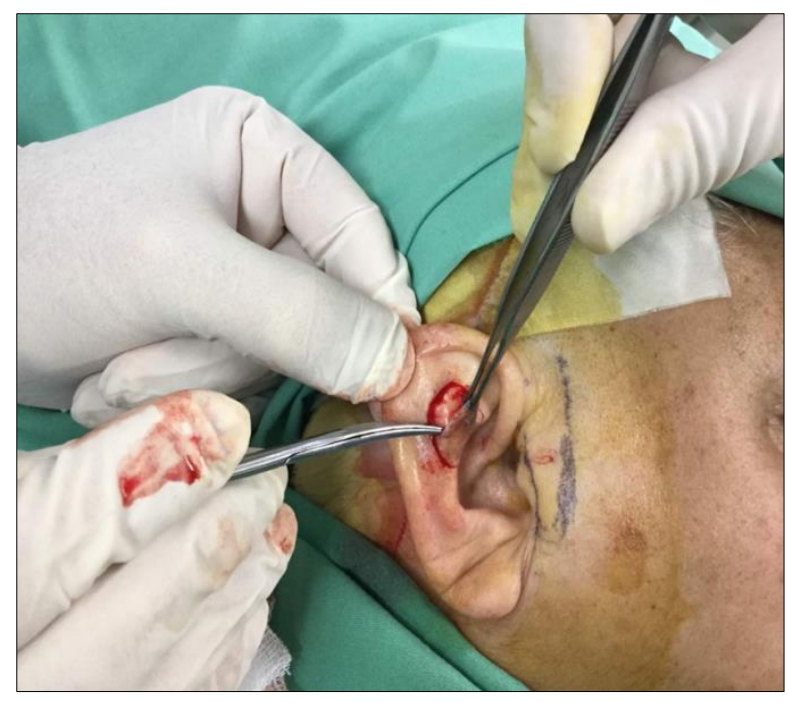

Figure 2 The lesion and the perichordrium removed through an excision

Biopsy was fixed in formalin and sent to a referral laboratory for histological preparation and examination. Final defect involved the region of the antihelix. A superiorly based flap was outlined along the hairless area of the preauricular region, and dissected from the distal margin, above the superficial temporal vessels. A tunnel was created under the cartilage of the anterior part of the helix (helicalcrus). The flap was transposed through the tunnel, over the fossa triangularis (superior antihelix), between the crus superius and crus inferius of the antihelix and laid atop the defect. We used thin skin sutures to fix the flap. We used a pressure bandage to prevent the occurrence of a hematoma, that remained in place for 24 hours (Figure 3 ).

Ten days later, the stitches removed safely, as the patient followed the aftercare instructions (Figure 4).

Both surgical incisions heal, so the final postoperative result was satisfactory (Figure 5). The histological examination result revealed a basal cell carcinoma, as expected. 
International Journal of Science and Research Archive, 2021, 03(02), 049-052

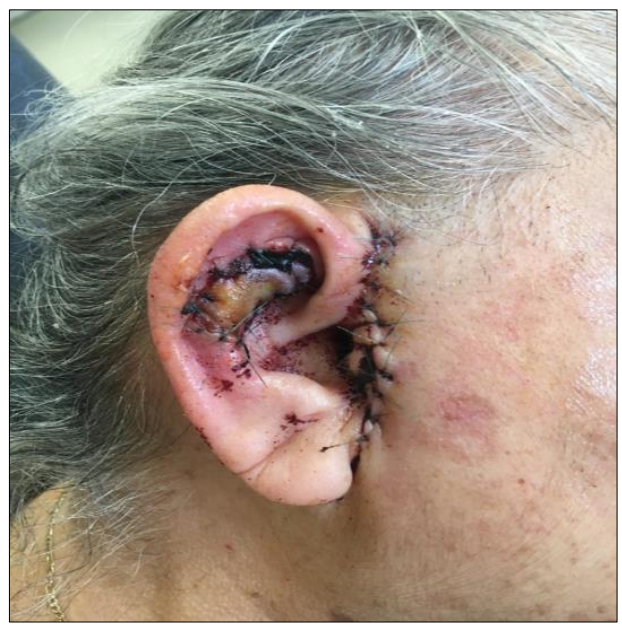

Figure 3 Postoperative result, 24 hours after the reconstruction

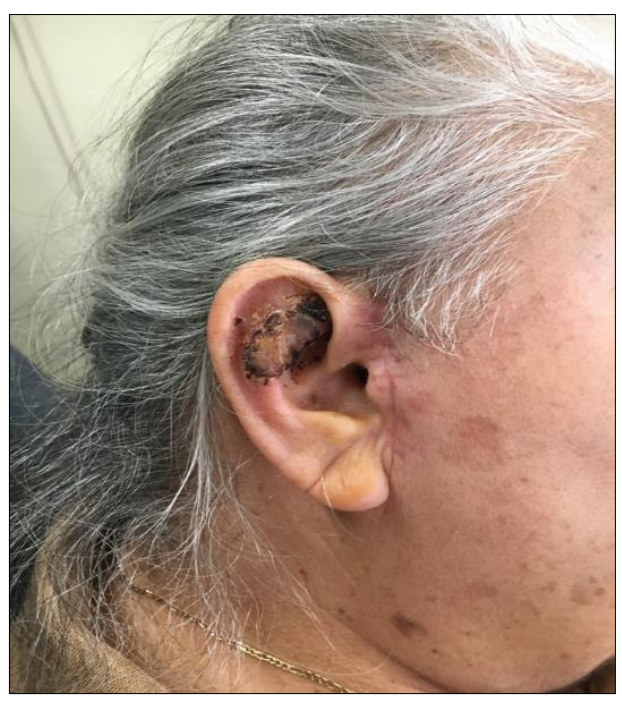

Figure 4 Postoperative result, after the stitches removal

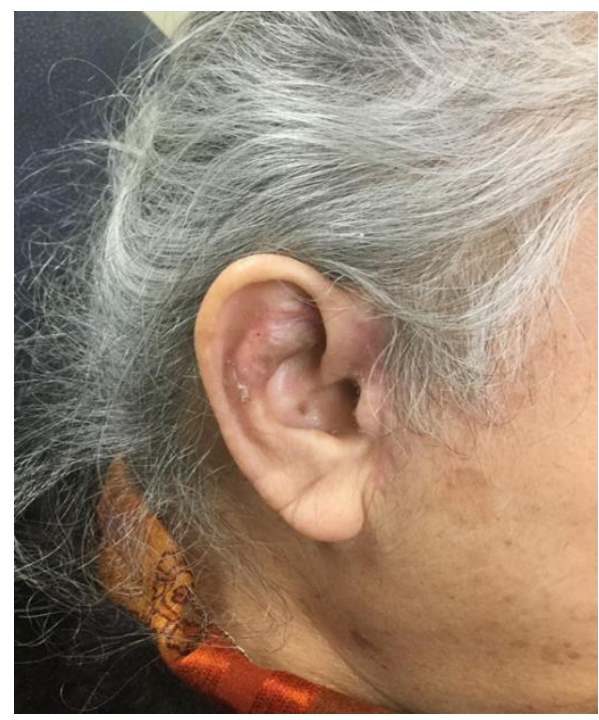

Figure 5 Final postoperative result, after surgical incisions heal 


\section{Discussion}

Basal cell carcinoma of the pinna is one of the commonest skin cancers of the head and neck area. Its localization can be anywhere on auricle, but usual areas of growth are the helix and antihelix. Many techniques proposed for the surgical excision of BCC of the pinna, using flaps to reconstruct the cartilage defects $[3,11,12]$. We used a pre- auricular flap so to reconstruct the antihelix defect of a 80-year-old woman BCC. We achieved complete coverage of the deficit remained after surgical excision of the lesion. The graft did not reject and the perfusion was effective.

\section{Conclusion}

The flap used for the reconstruction after a surgical incision of a skin cancer of the pinna, depends on the area that the lesion is located. We used a superiorly based pre- auricular flap and a cartilage tunnel to cover the antihelix defect. The postoperative results after removing the sutures were very satisfactory, both functionally and aesthetically. We believe that the use of pre- auricular flap is an appropriate method for skin lesions located in the antihelix of the pinna.

\section{Compliance with ethical standards}

\section{Disclosure of conflict of interest}

The authors declare no conflicts of interest regarding the publication of this paper.

\section{Statement of informed consent}

Informed consent was obtained from all individual participants included in the study.

\section{References}

[1] Bailin PL, Levine HL, Wood BG, Tucker HM. Cutaneous Carcinoma of the Auricular and Periauricular Region. Archives of Otolaryngology - Head and Neck Surgery. 1980; 106(11): 692-696.

[2] Silapunt S, Peterson SR, Goldberg LH. Squamous Cell Carcinoma of the Auricle and Mohs Micrographic Surgery. Dermatologic Surgery. 2005; 31(11): 1423-1427.

[3] Ahmad I, Gupta ARD. Epidemiology of basal cell carcinoma and squamous cell carcinoma of the pinna. The Journal of Laryngology \& Otology. 2001; 115(02).

[4] Majumdar A, Townend J. Helix rim advancement for reconstruction of marginal defects of the pinna. British Journal of Oral and Maxillofacial Surgery. 2000; 38(1): 3-7.

[5] McInerney NM, Piggott RP, Regan PJ. The trap door flap: A reliable, reproducible method of anterior pinna reconstruction. Journal of Plastic, Reconstructive \& Aesthetic Surgery. 2013; 66(10): 1360-1364.

[6] Jayarajan R. A versatile flap for reconstruction of partial pinna defects - The preauricular flap. JPRAS Open. 2017; 13: $49-52$.

[7] Reid RR, Lee MJ, Lewis VL. Postauricular Artery Island Flap for Subtotal Ear Reconstruction: Expanding Flap Versatility Based on Zones of Regional Perfusion. Plastic and Reconstructive Surgery. 2008; 121(4): 213e-214e.

[8] Levin BC, Adams LA, Becker GD. Healing by Secondary Intention of Auricular Defects After Mohs Surgery. Archives of Otolaryngology - Head and Neck Surgery. 1996; 122(1): 59-66.

[9] Thuile T, Larcher L, Gatscher B, Schwaiger K, Deluca J, Fallaha A, Eisendle K. Split-thickness skin grafting for reconstruction of auricular skin defects: a statistical analysis. JDDG: Journal Der Deutschen Dermatologischen Gesellschaft. 2018; 16(2): 163-173.

[10] Jackson IT, Milligan L, Agrawal K. The versatile revolving door flap in the reconstruction of ear defects. European Journal of Plastic Surgery. 1994; 17(3).

[11] Brouard M, Kaya G, Vecchietti G, Chavaz P, Harms M. Basal Cell Carcinoma of the Earlobe after Auricular Acupuncture. Dermatology. 2002; 204(2): 142-144.

[12] Levine HL, Bailin PL. Basal Cell Carcinoma of the Head and Neck: Identification of the High Risk Patient. The Laryngoscope. 1980; 90(6): 955-961. 